Wilfrid Laurier University

Scholars Commons @ Laurier

Physics and Computer Science Faculty

Publications

Physics and Computer Science

1988

\title{
Analog Simulation of Superconducting Loops Containing One or Two Josephson Junctions
}

James A. Blackburn

Wilfrid Laurier University, jabjabjab@cogeco.ca

Binrou Wu

Wilfrid Laurier University

H.J.T. Smith

University of Waterloo

Follow this and additional works at: https://scholars.wlu.ca/phys_faculty

\section{Recommended Citation}

Blackburn, James A.; Wu, Binrou; and Smith, H.J.T., "Analog Simulation of Superconducting Loops Containing One or Two Josephson Junctions" (1988). Physics and Computer Science Faculty Publications. 44.

https://scholars.wlu.ca/phys_faculty/44

This Article is brought to you for free and open access by the Physics and Computer Science at Scholars Commons @ Laurier. It has been accepted for inclusion in Physics and Computer Science Faculty Publications by an authorized administrator of Scholars Commons @ Laurier. For more information, please contact scholarscommons@wlu.ca. 


\title{
Analog simulation of superconducting loops containing one or two Josephson junctions
}

\author{
James A. Blackburn and Binruo Wu $\mathrm{Wu}^{\mathrm{a}}$ \\ Department of Physics and Computing, Wilfrid Laurier Universigy, Waterloo, Ontario, Canada \\ H.J.T. Smith \\ Department of Physics, University of Waterioo, Waterioo, Ontario, Canada
}

(Received 16 March 1988; accepted for publication 17 May 1988)

\begin{abstract}
Analog circuits are described which are capable of electronically simulating the static and dynamic behavior of superconducting loops containing one or two Josephson junctions when bias currents or magnetic fields are applied. Time-dependent fux entry into or out of the ring can, in either system, be observed by monitoring appropriate node voltages within the simulator circuits. The various dynamical modes observed in earlier numerical simulations are accurately reproduced. A theoretical analysis of the two-junction configuration identifies certain important criteria which determine which of these different states the system will adopt.
\end{abstract}

\section{INTRODUCTION}

The technology of superconducting devices based on the Josephson effect has now reached a rather mature state as evidenced by the commercial avaliability of a variety of instruments which utilize rf and de SQUIDs. Two recent discoveries have revived interest in the more fundamental properties of Josephson weak link circuits. The first is deterministic chaos, which seems to be an almost ubiquitous feature of nonlinear systems, and is known to appear in biased Josephson junctions ${ }^{1,2}$ and superconducting quantum interference devices. ${ }^{7}$ The second is the unexpected discovery of high $T_{c}$ superconducting materials; an advance which is likely to significantly broaden the scope of applications of weak link devices.

In attemping to predict the behavior of superconducting networks containing one or more Josephson elements, one invariably is faced with the task of solving nonlinear differential equations. With few exceptions, the method of choice has been to perform numerical simulations on digital computers. However an alternative-analog simulation-is availabie which offers the fiexibility associated with interactive, real-time processing. This approach was used, for example, by Bryant and Wiesenfeld ${ }^{4}$ in a recent sêdy of period doubling in a forced Duffing oscillator. In this paper an analog simulator is discussed which models the behavior of a superconducting ring intercupted by one or two Josephson junctions. The dynamics of bundles of fux quanta entering or leaving the loop are observed by monitoring appropriate node voltages within the simulation circuit.

The portion of our simulator which represents a Josephson junction is similar to an arrangement originally proposed by Magerlein ${ }^{5}$ and used subsequently by Kao et al.6,7 to study chaos. A nearly identical arrangenent was devised by Tuckerman ${ }^{8}$ for modeling double-junction interferoneters. In contrast to these circuits, however, our design, which has aiready been successfully applied to a study of chaos in

\footnotetext{
a) Visiting Scholar from Chengdu Institute of Radio Engineering, Chengdu, Sichuan, People's Republic of China.
}

coupled zero-capacitance weak links, ${ }^{9}$ does not require resetting logic for op amp saturation avoidance.

For the sake of completeness in this discussion, it should be noted that a second type of simulator due originally to Bak and Pedersen ${ }^{10}$ has also proved to work well in studies of single junctions, 1,12 double- and single-junction SQUDDs, ${ }^{13}$ microbridge interactions, ${ }^{14}$ and chaos in junctions with applied ac bias. ${ }^{15}$ In their approach, Bak and Pedersen modeled the quantum phase difference across a Josephson junction by the electronic phase difference between a voitage controlled oscillator and an external fixed frequency reference oscillator. As can be deduced from the literature already cited, the $\mathrm{Bak} / \mathrm{Pedersen}$ and Magerlein schemes each continue to have their proponents.

\section{ONE JUNCTHOH IN AOOP}

The system to be considered in this section consists of a superconducting ring interrupted by a single Josephson junction. The Josephson element is described by the resistively shunted junction model ( $R S . C$ ), with capacitance $C_{\text {, }}$ resistance $R$, and critical current $i_{c}$. The total loop inductance is $L$, and an applied magnetic field imposes an external fux $\phi_{x}$ on the ring. The $n$ et fux within the loop is denoted by 4. The derivation of the resulting differential equation for this configuration has been discussed elsewhere ${ }^{16,17}$; it is

$$
\Phi^{\prime \prime}+\beta \Phi^{\prime}+\gamma \sin (2 \pi \Phi)=\Phi_{x}-\Phi_{s}
$$

where $\Phi$ and $\Phi_{x}$ measure $\phi$ and $\phi_{x}$ in units of the fux quantum $\left(\phi_{0}\right), \beta=(1 / R) \sqrt{\ell} / C$ is the damping parameter, $\gamma=L i_{C} / \phi_{0}$, and time is measured in units of $\sqrt{L C}$.

The simulation circuit is shown in Fig. 1. The Josephson junction analog is synthesized from the combination of the operational amphifier $U_{1}$, resistor $R$, capacitance $C$, and the voltage-controlied oscillator (VCO) in series with $R_{C}$. The circuit for the $\mathrm{VCO}$ has already been described in detai1. ${ }^{\circ} \mathrm{Op}$ amp $U_{2}$ is a simple inverter while $U_{3}$ together with $R_{t}$ and $C_{L}$ serves to simulate the loop inductance. An analysis of the circuit based on the summing of currents at the inverting 


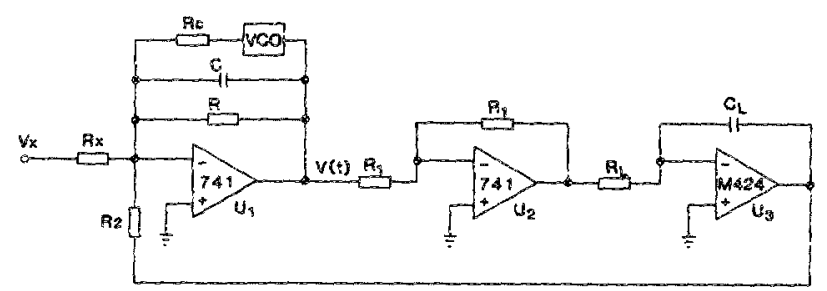

FIG. 1. Schematic diagram of the analog simulator circuit for a single Josephson junction in a superconolucting ring.

input of $U_{1}$ yields the following equation:

$$
\begin{gathered}
C \frac{d V}{d t}+\frac{1}{R} V+\frac{a}{R_{C}} \sin \left(2 \pi b \int V d t\right) \\
=-\frac{V_{x}}{R_{x}}-\frac{1}{R_{2} R_{L} C_{L}} \int V d t
\end{gathered}
$$

where $a$ is the amplitude of the sinusoidal VCO output and $b$ is a parameter specifying the $\mathrm{VCO}$ in units of $\mathrm{Hz}$ per volt.

If $\theta$ is defined as $k f(V d t)$, where $k$ is some constant to be determined, and time is normalized to units of $\sqrt{\left(\bar{R}_{2} \bar{R}_{L} \bar{C}_{L} C\right)}$, then Eq. (2) becomes

$$
\begin{aligned}
\theta^{\prime \prime} & +\left(\frac{1}{R} \sqrt{\frac{R_{2} R_{L} C_{L}}{C}}\right) \theta^{\prime}+\left(\frac{k a R_{2} R_{L} C_{L}}{R_{C}}\right) \\
& \times \sin \left(\frac{2 \pi b}{k} \theta\right)=-\left(k R_{2} R_{L} C_{L} V_{x} / R_{x}\right)-\theta
\end{aligned}
$$

which clearly corresponds to Eq. (1) provided $\Theta=\Phi$, $b / k=1$, and

$$
\begin{aligned}
& (1 / R) \sqrt{R_{2} R_{L} C_{L} / C}=\beta, \\
& k a R_{2} R_{L} C_{L} / R_{C}=\gamma
\end{aligned}
$$

and

$$
-k R_{2} R_{L} C_{L} V_{x} / R_{x}=\Phi_{x}
$$

The negative sign in (4c) implies that $V_{x}<0$ is required to simulate a positive applied bias flux.

There are considerably more variables appearing in the simulator circuit than the three $\left(\beta, \gamma, \Phi_{x}\right)$ required to fully specify the original fux-biased loop. One way of simplifying this situation is to recall that time in Eq. (3) is scaled to the interval $\tau=\sqrt{\left(R_{2} R_{L} C_{L} C\right)}$. Therefore values of $R_{2} R_{L} C_{Z}$, and $C$ can be chosen so that the waveforms to be observed on the simulator will occupy a total time interval which is experimentally "convenient."

Since the VCO parameters $a$ and $b$ remain fixed, it can be seen that for a given preset value of $\tau, \beta$ may be adjusted by means of $R, \gamma$ can be set by $R_{C}$, and $\Phi_{x}$ is controlled by the ratio $V_{x} / R_{x}$. This, then, is the prescription for choosing all of the circuit components in the simulator.

A series of experiments was carried out with $R_{2}=30$ $\mathrm{k} \Omega, R_{L}=4.17 \mathrm{M} \Omega, C_{L}=0.1 \mu \mathrm{F}$, and $C=3.67 \mathrm{nF}$; hence $\tau=6.78 \mathrm{~ms}$. For the VCO, $a=0.8 \mathrm{~V}$ and $b=1000 \mathrm{~Hz} / \mathrm{V}$. A $\gamma$ value of 1000 was selected by choosing $R_{C}=10 \mathrm{k} \Omega$, while different $\beta$ were obtained by varying $R$ throughout the range $1-100 \mathrm{k} \Omega$. Each run began by slowly increasing $V_{x}$ until, in the language of Ref. 16 , the critical bias fux was reached.
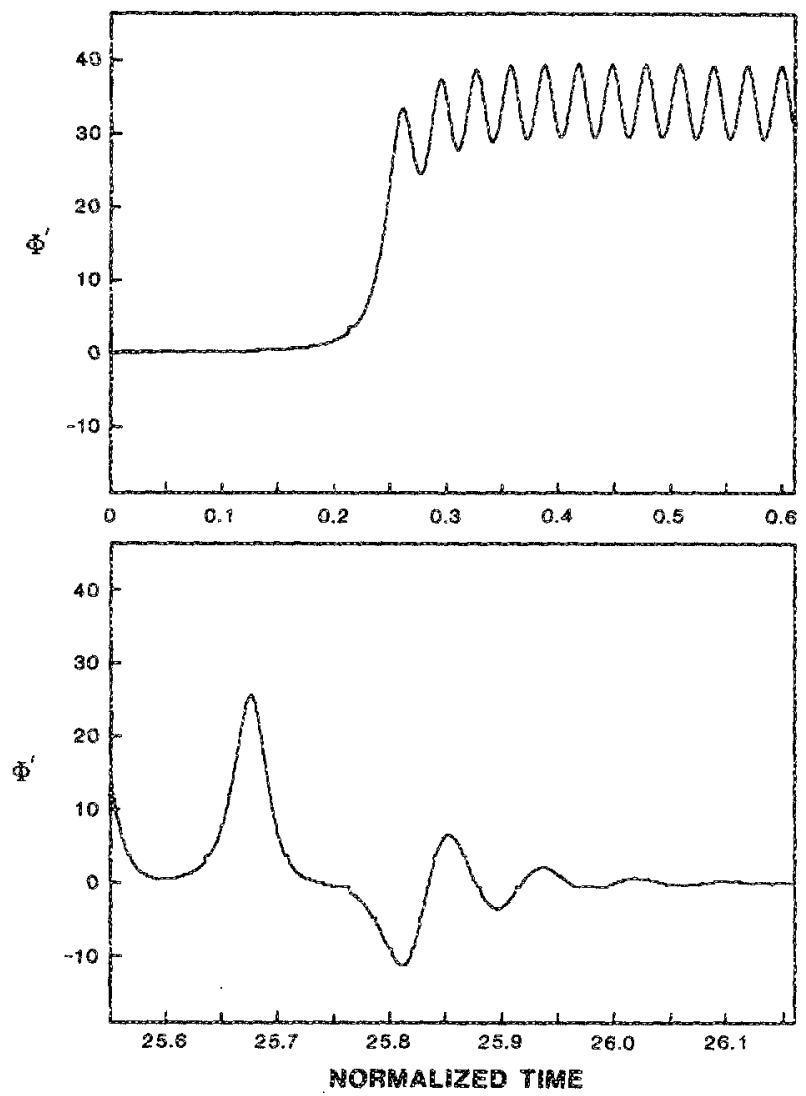

FIG. 2. Simulator voltage captured with a waveform recorder and rescaled to represent normalized hix velocity vs time. Parameters were $y=1000$ and $\beta=30$. The initial stage of fux entry is shown in the upper figure. The lower portion consists of the last quantum of the fux packet, followed by damped plasma oscillations.

The subsequent penetration of fiux quanta into the loop was observed on the simulator as a series of maxima and minima in the waveform appearing at the output of op amp $U_{1}$. From the definition of $\theta$ given earlier, it can be shown that $\Phi^{\prime}=\Theta^{\prime}=\left[k \sqrt{\left(R_{2} R_{L} \widehat{C_{L}} C\right)} V\right]$. This expression provides the numerical factor for converting observed voltages to dimensionless flux velocities. A Biomation model 2805 waveform recorder was used to capture the signals of interest.

A typical result is shown in Fig. 2. The capabilities of the waveform recorder made it possible to separately acquire magnified sections of $V(t)$ at the beginning and end of the flux transit sequence. A comparison with Fig. 1 of Ref. 16, which resulted from numerical solutions of the differential equation with an identicai value for $\gamma$ but slightly larger damping, confirns the quality of data which the simulator circuit is capable of generating.

The total number of flux quanta which enter the loop in any given situation can be obtained from the simulator by simply counting the number of peaks in the $V(t)$ waveform. In this manner, and by selecting various values for $R$, a plot of $\Phi_{\text {enter }}$ vs $\beta$ was obtained. These results are shown in Fig. 3 rogether with numerical simulation data which were presented originally in Ref. 16. Again, the agreement is excellent. 


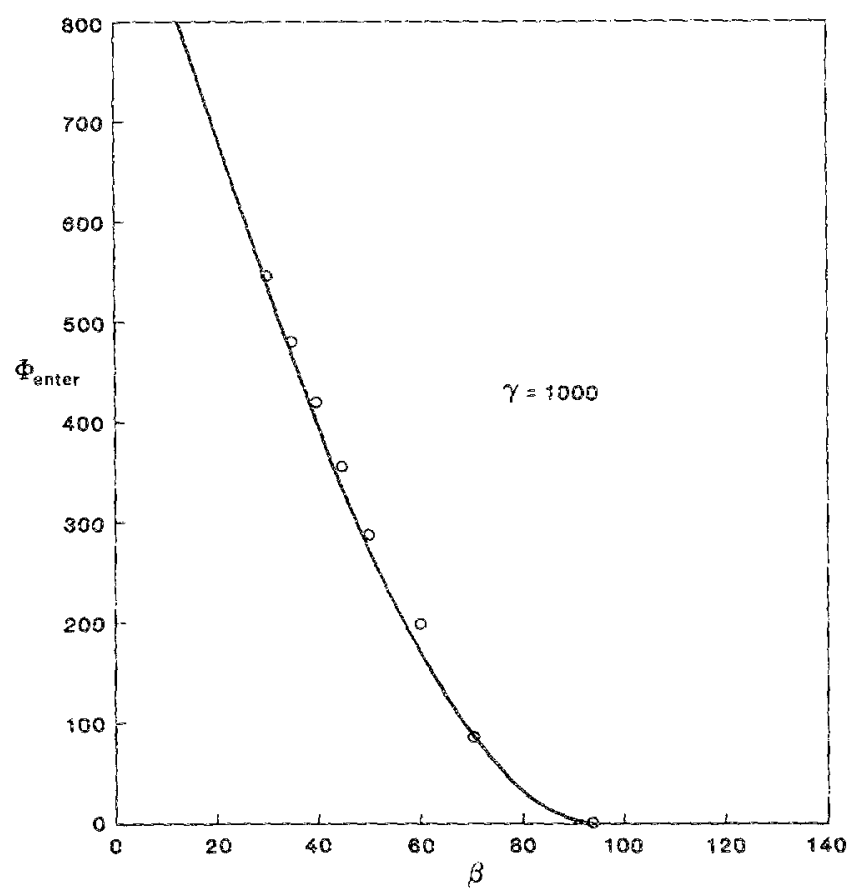

FIG. 3. A plot of the number of fux quanta entering the loop as a function of the damping parameter. The continuous line is from numerical simulations; data points are measured values obtained from the analog simulator.

\section{TWO IUNGTIONS IN LOOP}

Now consider the case of two identical Josephson junctions in an asymmetrically current-biased superconducting ring of total inductance $2 L$, as shown in Fig. 4. This system was studied by Blackburn and Smith ${ }^{18}$ who carried out numerical simulations of the coupled diferential equations which govern the individual junction phases $\varphi_{1}$ and $\varphi_{2}$ :

$\varphi_{1}^{\prime \prime}+\beta \varphi_{\mathrm{i}}^{\prime}+2 \pi \gamma \sin \left(\varphi_{1}\right)=2 \pi \alpha \gamma \vec{l}_{S}-\frac{1}{2}\left(\varphi_{1}-\varphi_{2}\right)$

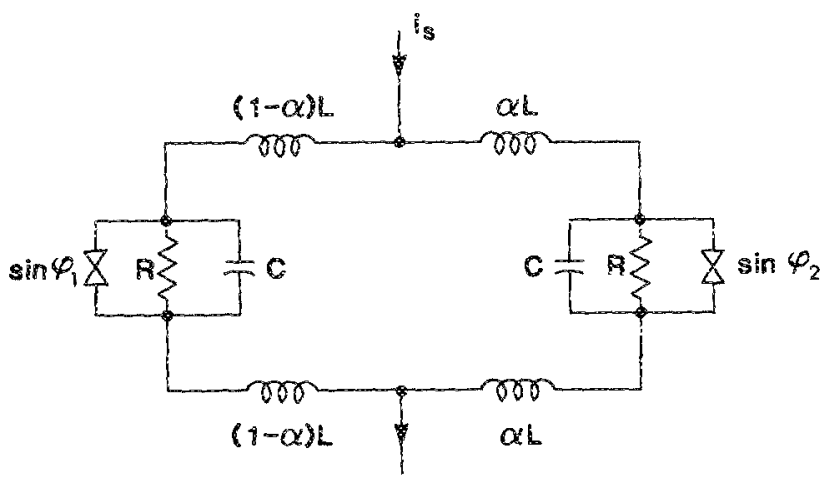

FIG. 4. Equivalent circuit of two Josephson junctions in a superconducting ring of inductance $2 L$. The bias current $I_{s}$ is asymmetrically applied to the loop.

and

$\varphi_{2}^{\prime \prime}+\beta \varphi_{2}^{\prime}+2 \pi \gamma \sin \left(\varphi_{2}\right)=2 \pi(1-\alpha) \gamma_{S}+\frac{1}{2}\left(\varphi_{1}-\varphi_{2}\right)$.

In the above, $\beta=(1 / R) \sqrt{(L / C)}, \gamma=l_{i} / \phi_{0}, l_{S}$ is an externally applied bias current expressed in units of the junction critical current $i_{c}, \alpha$ is a parameter specifying the relative location of the bias injection point, and time is normalized by the factor $\sqrt{(\bar{L} C)}$.

The simulator circuit for this interferometer is shown in Fig. 5. By asserting current conservation at the inverting inputs of op amps $U_{1}$ and $U_{2}$, we obtain

$$
\begin{gathered}
C \frac{d V_{B}}{d t}+\frac{1}{R} V_{1}+\frac{a}{R_{C}} \sin \left(2 \pi b \int V_{1} d t\right) \\
=-\frac{V_{x 1}}{R_{x 1}}-\frac{1}{R_{2} R_{I} C_{L}} \int\left(V_{1}-V_{2}\right) d t
\end{gathered}
$$

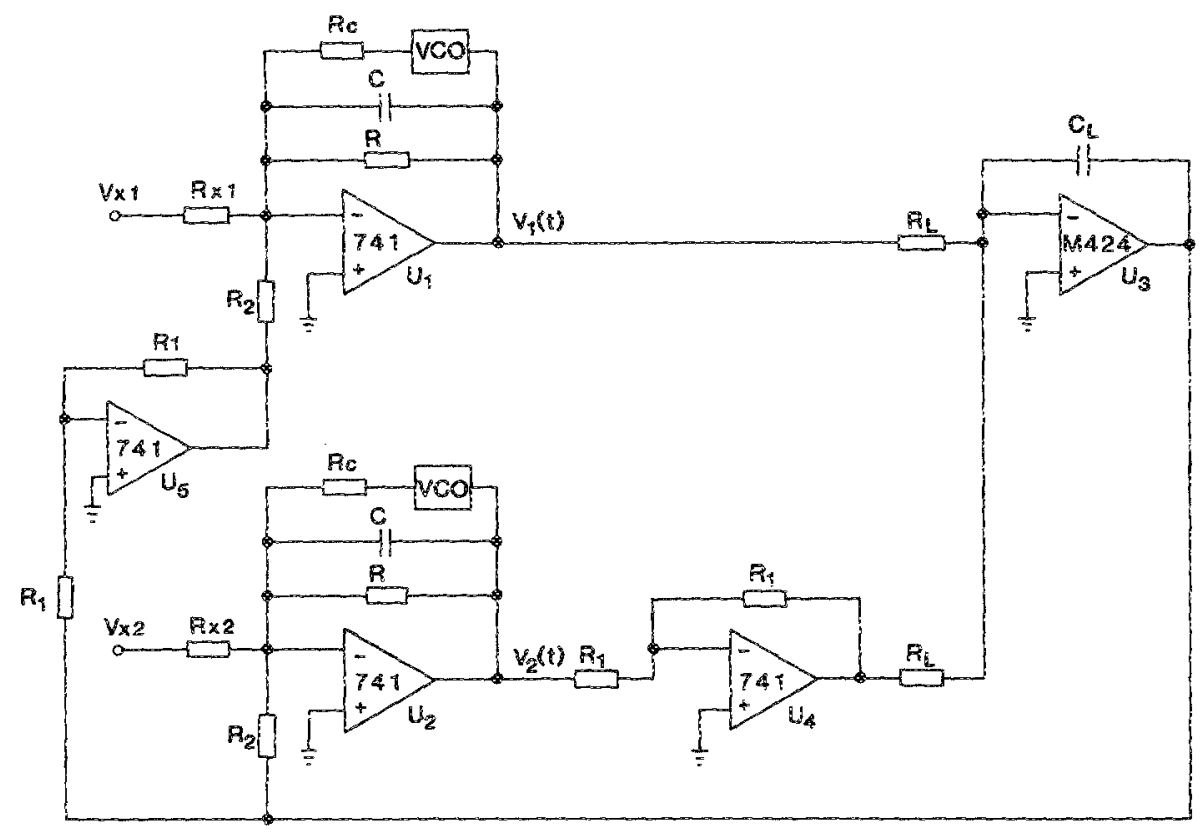

FIG. 5. Schematic diagram of the analog simulator circuit for the double-junction system. 
and

$$
\begin{gathered}
C \frac{d V_{2}}{d t}+\frac{1}{R} V_{2}+\frac{a}{R_{C}} \sin \left(2 \pi b \int V_{2} d t\right) \\
=-\frac{V_{x_{2}}}{R_{x_{2}}}+\frac{1}{R_{2} R_{L} C_{L}} \int\left(V_{1}-V_{2}\right) d t .
\end{gathered}
$$

Define $\theta_{1}=2 \pi k \delta\left(V_{1} d t\right)$ and $\theta_{2}=2 \pi k \delta\left(V_{2} d t\right)$, and normalize time to units of $\sqrt{R_{2} R_{L} C_{L} C / 2}$; then $(6 a)$ and (6b) become

$$
\begin{aligned}
\theta_{i}^{\prime \prime} & +\left(\frac{1}{R} \sqrt{\frac{R_{2} R_{L} C_{L}}{2 C}}\right) \theta_{i} \\
& +\left(\frac{\pi k \sigma R_{2} R_{L} C_{L}}{R_{C}}\right) \sin \left(\frac{b}{k} \theta_{i}\right) \\
& =\left(-\pi k R_{2} R_{L} C_{L} V_{x 1} / R_{x 1}\right)-\frac{1}{2}\left(\theta_{1}-\theta_{2}\right)
\end{aligned}
$$

and

$$
\begin{aligned}
\theta_{2}^{\prime \prime} & +\left(\frac{1}{R} \sqrt{\frac{R_{2} R_{L} C_{L}}{2 C}}\right) \theta_{2}^{\prime} \\
& +\left(\frac{\pi k a R_{2} R_{L} C_{L}}{R_{C}}\right) \sin \left(\frac{b}{k} \theta_{2}\right) \\
& =\left(-\pi k R_{2} R_{L} C_{L} V_{x 2} / R_{x 2}\right)+\frac{1}{2}\left(\theta_{1}-\theta_{2}\right) .
\end{aligned}
$$

Equations (7a) and (7b) correspond exactly to (5a) and ( $5 b$ ) provided $\theta_{1}=\varphi_{1}, \Theta_{2}=\varphi_{2}, b=k$, and

$$
\begin{aligned}
& (\hat{l} / R) \sqrt{R_{2} R_{L} C_{L} / 2 C}=\beta, \\
& \operatorname{kaR}_{2} R_{L} C_{L} / 2 R_{C}=\gamma, \\
& \left(1+\frac{V_{x z}}{V_{x 1}} \frac{R_{x 1}}{R_{x 2}}\right)^{-1}=\alpha,
\end{aligned}
$$

and

$$
-\frac{R_{C}}{a}\left(\frac{V_{x \mathrm{1}}}{R_{x 1}}+\frac{V_{x 2}}{R_{x 2}}\right)=I_{s}
$$

The numerical factor relating dimensionless time derivatives to the physically observed circuit voltages is given by

$$
\varphi^{\prime}=\left[\pi k \sqrt{\left(2 R_{2} R_{L} C_{L} C\right)}\right] \nu .
$$

Once again, a convenient approach is to select $R_{2}, R_{L}$, $C_{L}$, and $C$ such that the resulting time scale is appropriate for experimental observations. Having fixed these component values, $\beta$ is then set by $R, \gamma$ is determined by $R_{c}, \alpha$ is adjusted by the product $\left(R_{x 1} / R_{x 2}\right)\left(V_{x 2} / V_{x 1}\right)$, and finally $I_{S}$ is controlled by the sum $\left(V_{x 1} / R_{x 1}+V_{x 2} / R_{x 2}\right)$, subject to the constraint imposed by maintaining constant $\alpha$. For our circuit we chose $R_{x 1}=R_{x 2}=30 \mathrm{k} \Omega$ and only used $V_{x i}$ and $V_{x 2}$ to determine $\alpha$ and $I_{S}$. Another simple procedure would be to use a single bias supply $\left(V_{x}=V_{x 1}=V_{x 2}\right)$ and control $\alpha$ through the ratio of the resistors $R_{x 1}$ and $R_{x 2}$.

In experimental runs the output of the integrator $\mathrm{U}_{3}$ was momentarily grounded-this was equivalent to clearing the loop of any initially trapped flux. $V_{x 1}$ and $V_{x 2}$ were then slowly increased (at a fixed ratio to preserve constant $\alpha$ ) until one of the junctions first reached its critical current and a dynamical fux state was entered. The time derivatives of the junction phases were observed during this interval by

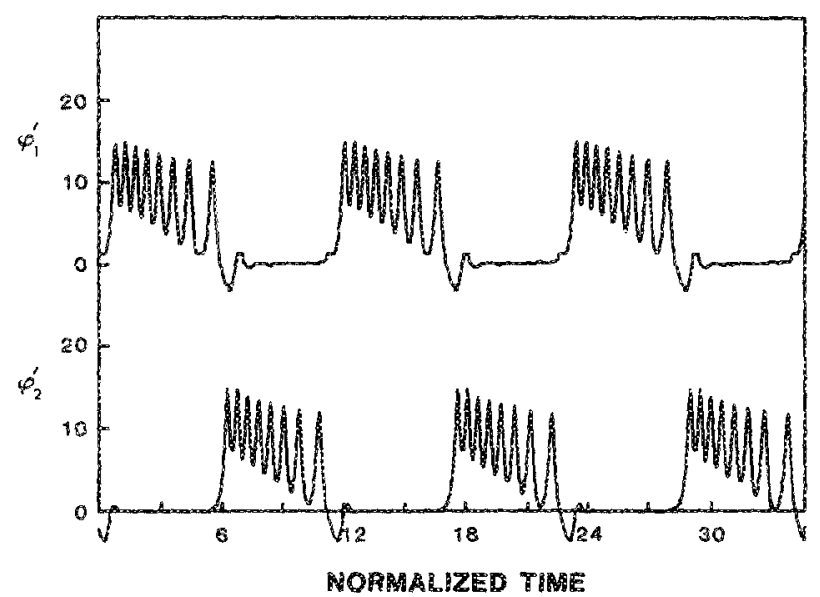

FIG. 6. Analog simulator output, suitably rescaled, representing the two junction voltages for $\gamma=10, \alpha=0.6$, and $\beta=4.5$.

monitoring the outputs of op amps $U_{1}$ and $U_{2}$ with the waveform recorder.

It was shown in Ref. 18 that, for fixed $\gamma$, three distinct types of behavior occur, depending on the value of the damping parameter. For $\beta$ greater than some number $\beta_{1}$, all flux quanta will move only through one of the two junctions and the system will act as if it contained only a single weak link. For $\beta$ less than a second value $\beta_{2}$, the loop will initially fll through this same junction, but the process will eventually be replaced by one in which a dynamical equilibrium exists with single quanta alternately entering the loop through the first junction and leaving the loop through the second. This switching state will continue as long as the external bias current is maintained. In the intermediate region, $\beta_{2}<\beta<\beta_{1}$, packets of $n$ quanta enter the loop through one junction and then leave the loop through the other junction, with fill-andempty cycles endlessly repeating.

All of the above processes have been observed on the simulator. An example of the intermediate state is illustrated in Fig. 6 where, for $\gamma=10$ and $\beta=4.5$, bundles of eight

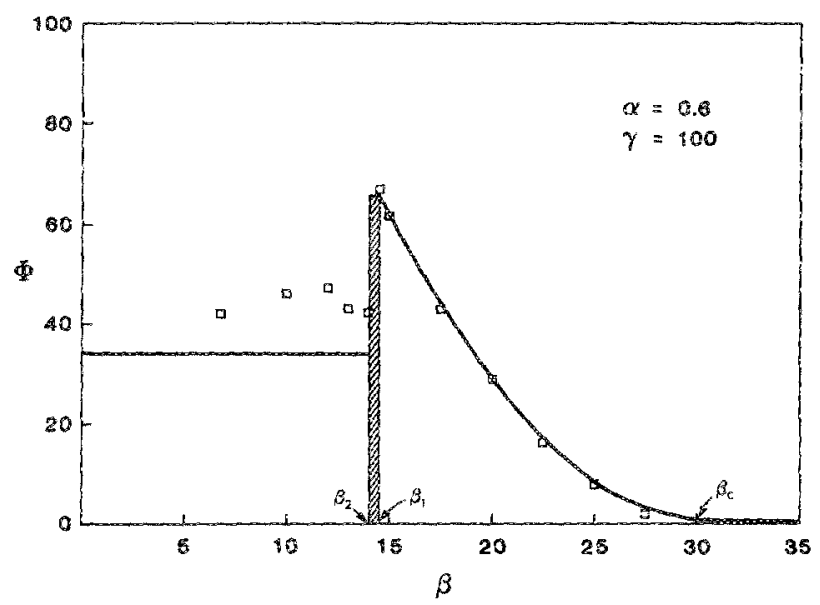

FlG. 7. Net fiux in the double-junction loop following the first bias current induced breakdown as a function of damping parameter. Continuous curves are from numerical simulations; data points were obtained from the analog simulator. 
"quanta" alternately enter and leave the ring. These results are in excellent agreement with those represented in Fig. 4 of Ref. 18. Data from a number of experimental runs with $\gamma=100$ are plotted in Fig. 7 and, again, quantitative agreement with the corresponding figure in Ref. 18 is very good, with the exception perhaps of the low damping regime where errors probably resulted from imprecise matching of the components representing the pair of junctions.

\section{DISCUSSION}

Some insight into the physical meaning of $\beta_{1}$ can be gained from the following analysis. Suppose $\beta \geqslant \beta_{1}$, and to facilitate the discussion, choose the particular value $\alpha=0.6$ (the conclusions will in no way depend on this specific choice). Imagine the bias current in Fig. 4 being increased until a breakdown to a dynamic state is about to occur. At this moment, the loop still contains essentially zero quanta, with $I_{J !}=0.6 I_{S}$ fowing through the left branch containing junction 1 , and $I_{\mathrm{J} 2}=0.4 I_{\mathrm{S}}$ passing through junction 2 . However, since breakdown is imminent at junction $\downarrow$, which is carrying the greater current, $I_{11}$ must equal 1 (currents have been normalized to the critical vahue $i_{c}$ ). Hence $I_{S}=1 / 0.6$ and so junction 2 carries $I_{32}=0.4 / 0.6=2 / 3$. An infinitesimal increment in $I_{S}$ will induce breakdown, following which a total of $n$ quanta will enter the loop. The bias current will continue to be divided between the two branches as before, but a circulating loop current $I_{L}$ will now also be present:

$$
n=2 \gamma I_{2} \text {. }
$$

The superposition of the loop current with the bias components in each branch resuits in a net current in junction 1 of $(1-n / 2 \gamma)$, and in junction 2 of $(2 / 3+n / 2 \gamma)$. The number $n$ depends on the damping parameter $\beta$ : it is quite small for large $\beta$, but conversely becomes large at smali $\beta$, and in fact ultimately tends towards the value $2 \gamma$.

Define $\beta_{1}$ as the damping for which $n=N=\frac{1}{3}(2 \gamma)$. In this particular case, junction 1 will carry $1-\frac{1}{3}=2 / 3$, while the current in junction 2 will be $\frac{2}{3}+\frac{1}{3}=1$. In other words, the conditions on the two junctions are exactly the reverse of their prebreakdown values.

Because the bias $I_{S}$ remains at its previous level, which was sufficient to initiate a breakdown at junction 1, it will now force a new sequence to begin just as junction 1 is terminating its activity with damped piasma oscillations, but this time the fux packet containing $N$ quanta will exit the loop via junction 2 . These arguments explain why such fill-andempty cycles occur in the vicinity of $\beta_{i}$. The identification of junction 1 (as specified in Fig. 4) as the one through which fux quanta first enter the loop is applicable for $\alpha>0.5$, in which case a generai expression for $N$ is

$$
N=2 \gamma(2-1 / \alpha) \text {. }
$$

For $\alpha=0.6$ and $\gamma=100$, this give $N=67$, in excellent agreement with the data presented in Fig. 7. For $\alpha=0.6$ and $\gamma=10$, Eq. (9) predicts $N=7$; the observed value indicated in Fig. 8 is eight quanta.

It also follows that if $M=\gamma(2-1 / \alpha)$ quanta reside in the loop, then $I_{51}$ will exactly equal $I_{32}$. It is this symmetry

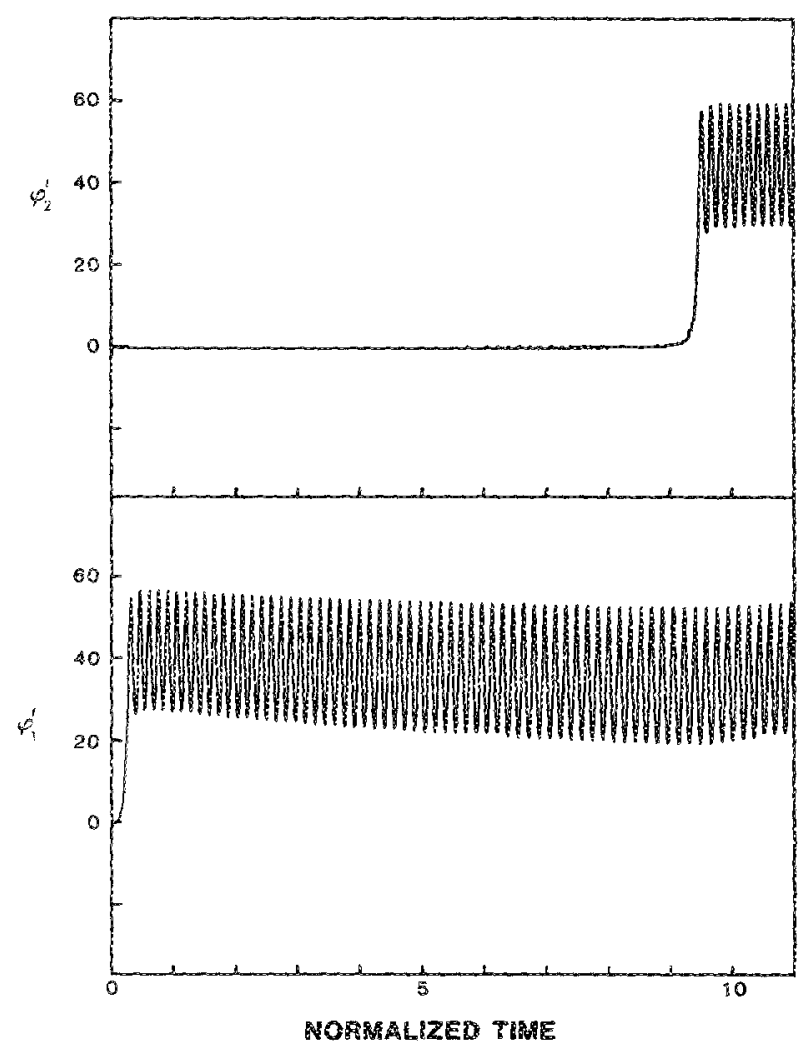

FIG. 8. Junction voltages observed on the analog simulator for $a=0.6$, $\beta==13.96$, and $\gamma=100$. This illustrates the onset of activity at both junctions. When the voltages eventually become synchronized, a dynamic equilibrium is established in which singie quanta alternately enter and exit the loop.

which characterizes the region below $\beta_{2}$ where the junctions alternate in passing single quanta in and out of the loop. Note that $M=N / 2$, and so the dynamic equilibrium of this switching mode maintains just one half of the flux value which occurs at $\beta_{1}$. This fact was deduced on emperical grounds in a previous publication. ${ }^{18}$

The other boundary (at $\beta_{2}$ ) of the fill-and-empty zone may be interpreted in the following manner. At $\beta$, the exchange of roies between junction 1 and junction 2 is induced by the initial transfer of precisely $N$ quanta into the ring. Suppose a smaller $\beta$ is chosen and the bias current is increased until junction 1 breaks down. As soon as the first $N$ quanta enter the loop, junction 2 will reach its critical current. Because of the low damping, junction 1 will still remain active and so the loop will simultaneously lose quanta through junction 2 and gain quanta through junction 1 , but at different rates-with losses exceeding gains. This type of behavior was observed on the simulator and an example is shown in Fig. 8 for $\alpha=0.6$ and $y=100$. The reduction in the number of quanta in the loop will continue until the rates just balance and the switching state referred to above is established. Therefore $\beta_{2}$ is that value of damping for which $N+1$ quanta would first enter the ring through junction 1 . In Ref. 16 it was shown that a "universal" curve for fux entry could be constructed by plotting $\Phi_{\text {enter }} / \gamma$ vs $\beta / \beta_{c}$, where $\beta_{c}$ is a constant times $\sqrt{\gamma}$. Such a curve for a loop of inductance $2 l$ is reproduced in Fig. 9 , to which has been 


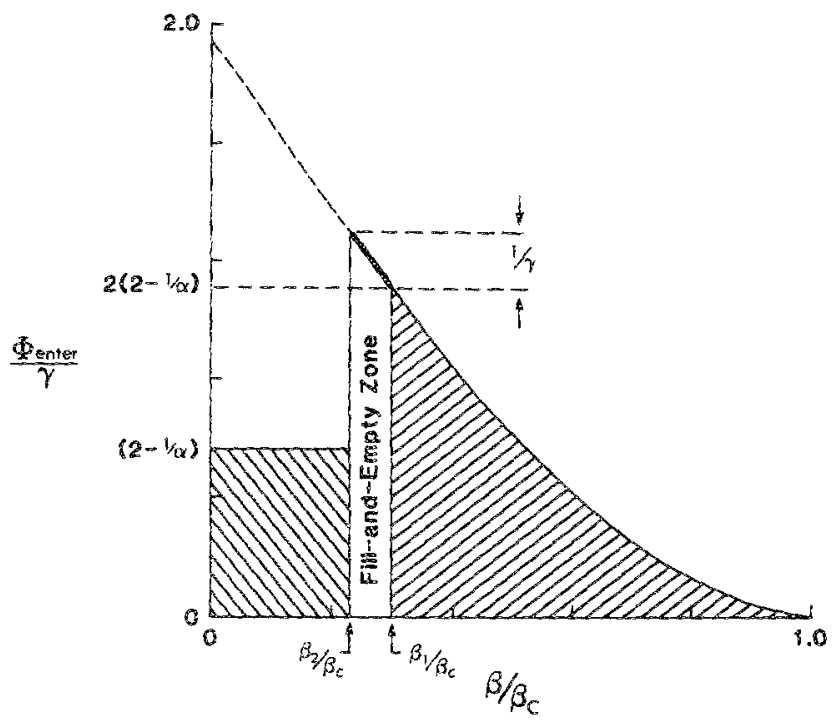

FIG. 9. Plot of the universal characteristic: $\Phi_{\text {enter }} / \gamma$ vs normalized damping. For a loop containing two Josephson junctions, a current induced breakdown at $\beta_{1}$ will lead to a fill-and-empty mode with bundles of $2(2-1 / \alpha)$ flux quanta alternately entering and leaving the loop.

added a hypothetical $\beta$, and its associated $N$. Because the ordinate is labeled in units of $\gamma$, an increase in flux to $N+1$ corresponds to a vertical shift of $1 / \gamma$. It is readily apparent from the figure that large $\gamma$ will lead to relatively small intervals along the normalized $\beta$ axis, and vice versa. In fact if we treat the $\Phi_{\text {enter }} / \gamma$ vs $\beta / \beta_{c}$ characteristic as approximately linear in the region of interest, then $\left(\beta_{1}-\beta_{2}\right) / \beta_{c}$ would vary as $1 / \gamma$. However, because $\beta$ itself is proportional to $\sqrt{\gamma}$, the net dependence should be $\left(\beta_{1}-\beta_{2}\right) \propto 1 / \sqrt{\gamma}$. A conjecture to this effect was made in Ref. 18. To see how this relationship could be applied, consider the fact that both nimerical and analog simulations yielded $\left(\beta_{1}-\beta_{2}\right)=0.6$ for $a=0.6$ and $\gamma=10$. From this value, we may then predict that $\left(\beta_{1}-\beta_{2}\right)$ should be 0.19 and 0.06 for $\gamma=100$ and 1000 , respectively. Estimates of corresponding widths of the fill-and-empty zone, based on simulation data, were 0.3 and 0.1 ; this level of agreement is quite consistent with the approximation which has been invoked.

Finally, we note that it should be possible to enter the fll-and-empty mode with values of the damping parameter greater than $\beta_{1}$. The process may be illustrated with the following example. Let $\alpha=0.6$ and $\gamma=100$ and raise the bias current $I_{S}$ from zero to 1.667. At this moment the net junction currents will be $I_{J_{1}}=1.0$ and $I_{32}=0.667$; junction 1 will therefore break down and fiux will enter the loop. Suppose the damping is such that $\Phi_{\text {enter }}=25$ so that after a steady state has been achieved 25 quanta will reside in the loop. Now let $I_{S}$ be increased further still to the value 1.875 . The junction currents are now found to be $I_{s_{1}}=1.0$ and $I_{32}=0.875$. Junction 1 will, consequently, break down a second time and an additional 25 quanta will be admitted into the loop. When a steady state is again established, the junction currents are $I_{31}=0.875$ and $I_{52}=1.0$. Note that the two junctions have now exactly reversed roles and so the fill-and-empty mode will appear. However, because the fux

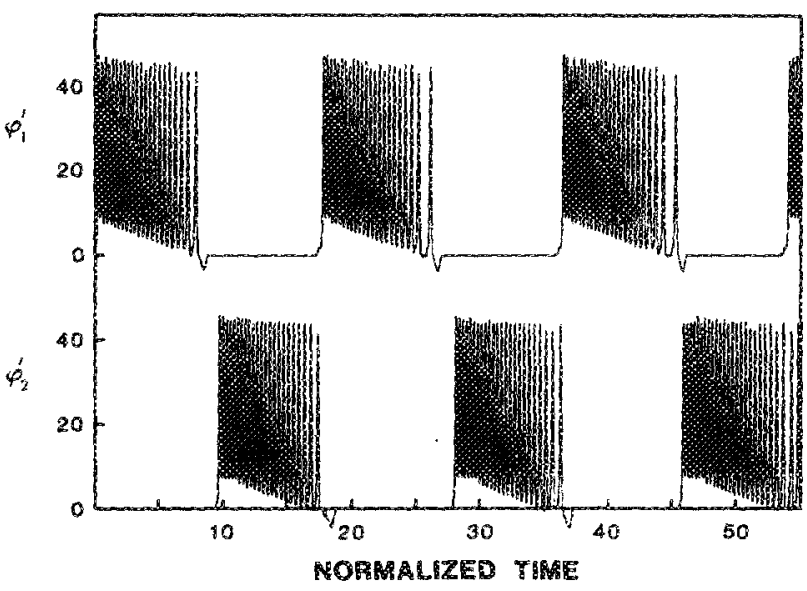

FIG. 10. Recorded waveforms confirming the existence of a fill-and-empty mode which occurs after second bias induced breakdown for $\alpha=0.6$, $\beta=20.3$, and $\gamma=100$.

bundles consist of 25 quanta in this situation, the alternating action of the two junctions will result in a net fiux which varies between 50 and 25 . This example shows how, for the particular values of alpha and gamma selected, a two-step process can lead to the fill-and-empty mode. If $\beta$ had been such that $\Phi_{\text {enter }}$ was not quite 25 , then exact role reversal would not occur after the second breakdown and the system would, either immediately or following a third current-induced breakdown, enter a switching state with single quanta entering and leaving the loop. In this case, an average fux of 37.5 would be maintained.

An experiment was carried out on the analog simulator to test the above hypothesis. Circuit components were selected so that $\beta=20.3$ and $\gamma=100$. The bias voltages $V_{x 1}$ and $V_{x z}$ were increased until the system had passed the first breakdown, and just reached its second critical condition. At this point, as shown by the recorded waveforms in Fig. 10, bundles of approximately 25 fiux quanta were seen to be transferred in and out of the loop. This observation confirms the existence of a two-step scenario for reaching the fill-andempty mode.

The question arises as to whether it is possible to enter the fill-and-empty mode from other values of $\beta>\beta_{1}$. Consider now arbitrary $\alpha$ and $\gamma$, and for the $\beta$ in question, assume each induced breakdown causes $m$ quanta to be transferred. Suppose $(k-1)$ critical increments in $I_{S}$ have already been forced and the system is still in equilibrium with $(k-1) m$ quanta in the loop. The next breakdown will be induced by a bias current ${ }^{18}$ :

$$
I_{\mathrm{SC}}=[1+(k-1) m / 2 \gamma] / \alpha \text {. }
$$

At this moment one finds the junction currents to be

$$
I_{51}=1.0
$$

and

$$
I_{32}=(1-\alpha) / \alpha+(k-1) m / 2 \alpha \gamma .
$$

Hence $m$ additional quanta enter the loop through junction 1. When this activity ceases, the loop contains $k m$ quan- 
ta, and the total junction currents are

$$
I_{51}=(1-m / 2 \gamma)
$$

and

$$
I_{s 2}=(1-\alpha) / \alpha+(m / 2 \gamma)[(k-1)+\alpha] / \alpha .
$$

From these expressions it can be shown that the two junctions have exactly exchanged roles in the course of this fux transfer, with $I_{y 2}$ now reaching unity, provided

$$
m=2 \gamma(2 \alpha-1) /[(k-1)+\alpha] \text {. }
$$

For $\alpha=0.6, y=100$, and $k=2$, Eq. (10) yields $m=25$-the value employed in the example discussed above. The fact that $m$ must be an integer eliminates many, if not most, $k$ values. For instance $k=3$ results in $m=15.38$ but neither $m=15$ nor $m=16$ will access the fill-and-empty mode in three jumps; instead the system will stabilize eventually in the switching mode.

\section{CONCLUSIONS}

Measurements performed on the analog simulators have demonstrated the accuracy with which these circuits model the behavior of superconducting loops containing one or two Josephson junctions. The parameters which completely specify the systems of interest are easily adjusted by varying either resistors or bias voltages. Because the coupled nonlinear differential equations governing the two junction configuration correspond to the mathematical description of a pair of torque-driven interacting pendulums, an analog simulator can also provide a means of probing the motion of this type of oscillator. Such an approach to studying nonlin- ear mechanical systems could be a useful alternative to numerical analysis, and could facilitate the exploration of their compiex dynamical states.

\section{ACKNOWEDGRENTS}

Yang Zhou-jing designed the portion of the simulator which models individual Josephson junctions. Financial support was provided by the Natural Sciences and Engineering Research Council of Canada.

'R. L. Kautz and R. Monaco, J. Appl. Phys. 57, 875 (1985).

${ }^{2}$ M. lansiti, Qing Hu, R. M. Westervelt, and M. Tinkham, Phys. Rev. Lett. 55, $746(1985)$.

${ }^{3}$ R. K. Ritala and M. M. Salomaa, Phys. Rev. B 29, 6143 (1984)

P. Bryant and K. Wiesenfeld, Phys. Rev. A 33, 2525 (1986).

5. H. Mageriein, Rev. Sci. Instrum. 49, 486 (1978).

'Y. H. Kao, J. C. Huang, and Y. S. Gou, J. Low Temp. Phys. 63, 287 (1986).

'Y. H. Kao, J. C. Huang, and Y. S. Gou, Phys. Rey. A 34, 1628 (1986).

'D. B. Tuckerman, Rev. Sci. Instrum. 49, 835 (1978).

${ }^{9}$ B. Wu, Y. Zhou-jing, I. A. Blackburn, S. Vik, H. J. T. Smith, and M. A. H. Nerenberg, Phys. Rev. B 37, 3349 (1988).

${ }^{\circ}$ C. K. Bak and N. F. Pedersen, Appl. Phys. Lett. 22, 149 (1973).

"D. E. Prober, S. E. G. Slusky, and R. W. Henry, and E. D. Jackel, J. Appl. Phys. 52, 4145 (1981).

${ }^{12}$ R. W. Henry, D. E. Prober, and A. Davidson, Am. J. Phys. 49, 1035 (1981).

${ }^{13}$ R. W. Henry and D. E. Prober, Rev. Sci. Instrum. 52, 902 (1981).

${ }^{14}$ R. Escudero and H. J. T. Smith, J. Appl. Phys. 56, 3271 (1984).

${ }^{15}$ D. DHumieres, M. R. Beasley, B. A. Huberman, and A. Libchaber, Phys. Rev. A 26, 3483 (1982).

"H. J. T. Smith and I. A. Blackburn, Phys. Rev. B 12, 940 (1975).

"J. A. Blackburn and H. 5. T. Smith, Phys. Rev. B 35, 1659 (1987).

"'s. A. Blackburn and H. J. T. Smith, J. Appl. Phys. 69, 2452 (1978). 